\title{
High-resolution Ammonia Mapping of the Protostellar Core Cha-MMS1
}

\author{
Miikka Väisälä ${ }^{1}$, Jorma Harju ${ }^{2}$, Maarit Mantere ${ }^{1}$, Oskari Miettinen ${ }^{1}$ \\ and Malcolm Walmsley ${ }^{3,4}$ \\ ${ }^{1}$ Department of Physics, University of Helsinki, \\ P.O. Box 64, FI-00014, Helsinki, Finland \\ email: miikka.vaisala@helsinki.fi \\ ${ }^{2}$ Finnish Centre for Astronomy with ESO, University of Turku, \\ Väisäläntie 20, FI-21500, Piikkiö, Finland \\ ${ }^{3}$ Dublin Institute for Advanced Studies, 10 Burlington Road, Dublin 4, Ireland \\ ${ }^{4}$ INAF - Osservatorio Astrofisico di Arcetri, \\ Largo Enrico Fermi 5, I-50125, Firenze, Italia
}

\begin{abstract}
The nearby protostellar core Cha-MMS1 has been mapped in the $\mathrm{NH}_{3}(1,1)$ line and the $1.2 \mathrm{~cm}$ continuum using the Australia Telescope Compact Array, ATCA. In addition, observations from Spitzer Space Telescope and Herschel Space Observatory are used to help the interpretation. An elongated condensation with a maximum length of $9000 \mathrm{AU}$ is seen in ammonia. The condensation has a clear velocity gradient directed perpendicularly to the axis of elongation. The gradient can be interpreted as rotation around this axis. We suggest that the observed ammonia structure delineates a rotating envelope and dense gas entrained by a very young protostellar outflow.
\end{abstract}

Keywords. stars: formation — ISM: clouds - ISM: jets and outflows

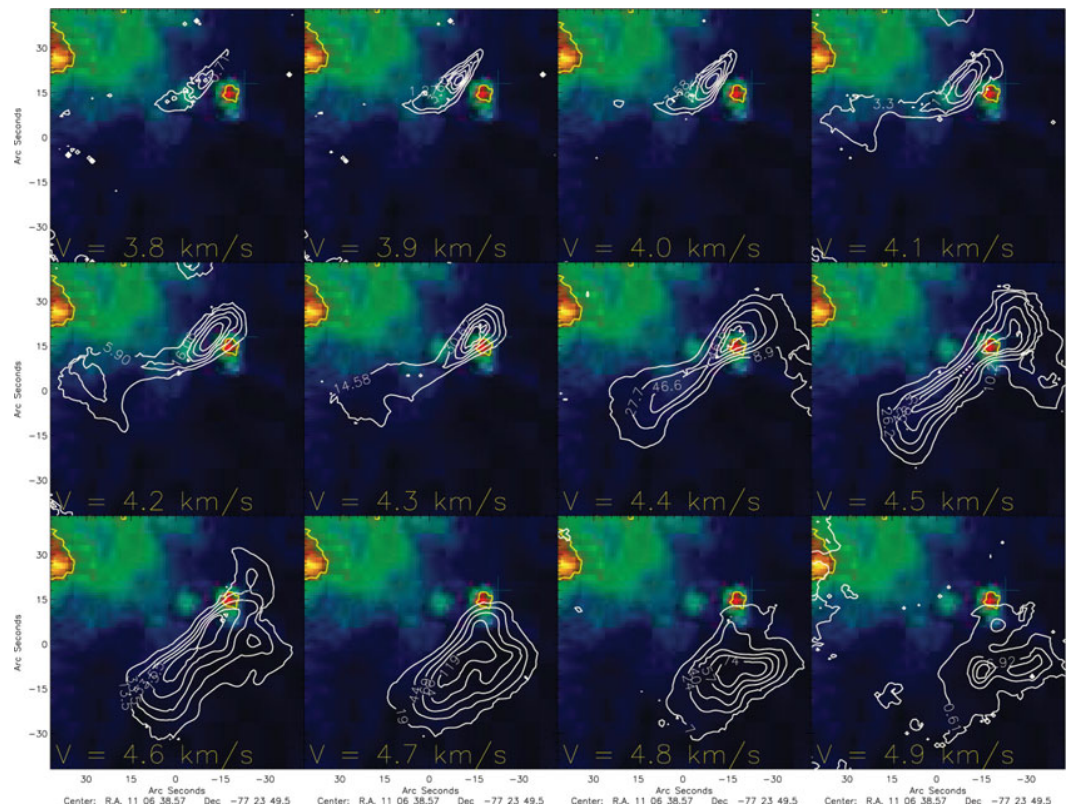

Figure 1. Velocity channel images of $\mathrm{NH}_{3}$. The color map shows Spitzer $24 \mu \mathrm{m}$ continuum. The compact source near the ammonia maximum represents dust heated by an embedded protostar. 